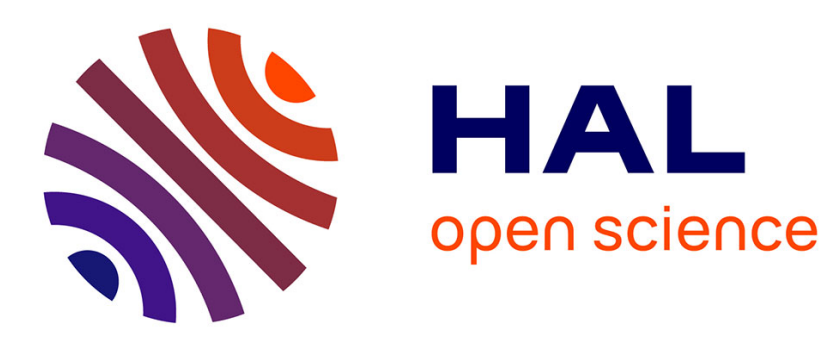

\title{
Le concept de tragicomedia et la question du genre dans quelques paratextes espagnols (XVIe et XVIIe siècles)
}

Christophe Couderc

\section{To cite this version:}

Christophe Couderc. Le concept de tragicomedia et la question du genre dans quelques paratextes espagnols (XVIe et XVIIe siècles). Littératures classiques, 2014, Préface et critique: le paratexte théâtral en France, en Italie et en Espagne (XVIe-XVIIe siècles) (83), pp.195-216. 10.3917/licla.083.0195 . hal-01543858

\section{HAL Id: hal-01543858 \\ https://hal.parisnanterre.fr/hal-01543858}

Submitted on 8 Nov 2018

HAL is a multi-disciplinary open access archive for the deposit and dissemination of scientific research documents, whether they are published or not. The documents may come from teaching and research institutions in France or abroad, or from public or private research centers.
L'archive ouverte pluridisciplinaire HAL, est destinée au dépôt et à la diffusion de documents scientifiques de niveau recherche, publiés ou non, émanant des établissements d'enseignement et de recherche français ou étrangers, des laboratoires publics ou privés. 


\section{Christophe Couderc}

\section{LE CONCEPT DE TRAGICOMEDIA ET LA QUESTION DU GENRE DANS QUELQUES PARATEXTES ESPAGNOLS (XVIE ET XVIIE SIÈCLES)}

La question de la taxinomie, ou le « problème de la classification ${ }^{1}$ » du théâtre espagnol du Siècle d'or a généré une bibliographie critique particulièrement abondante ${ }^{2}$. La masse et la variété des œuvres écrites pour leur représentation en Espagne, au XVle et au XVII siècles, incitent en effet spontanément l'observateur à tenter d'établirune classification, ne fût-ce qu'aux fins pragmatiques de la description d'un corpus fort de plusieurs milliers de pièces ${ }^{3}$. Mais l'ampleur du corpus n'est pas la seule difficulté dont doivent tenir compte les propositions taxinomiques ; celles-ci doivent également s'accommoder de la pauvreté conceptuelle, voire de l'absence de textes dans lesquels pourrait se trouver formulée une théorie du théâtre du Siècle d'or contemporaine de son développement ${ }^{4}$.

1. «El problema de la clasificación de los géneros » : titre du premier chapitre de M. Newels, Los géneros dramáticos en las poéticas del siglo de oro, Londres, Tamesis, 1974, p. 41-53.

2. Voir la synthèse de M. Vitse dans J. Huerta Calvo (dir.), Historia del teatro español, Madrid, Gredos, 2003, vol. 1, p. 745-748 et p. 750-755 (bibliographie).

3. Les estimations oscillent entre 10000 pièces sur un siècle et demi, ou 15000 sur un siècle. Le corpus textuel conservé compte tout de même plus de 5000 titres et indique assez bien à lui seul que, dans le monde de la comedia, tout est démesure.

4. «La théorie dramatique espagnole peut être dite pauvre, conjoncturelle, fragmentaire et asystématique », signale Marc Vitse, qui souligne «la brièveté, l'incomplétude et le pragmatisme » de ces textes théoriques ainsi que leur « éparpillement, émiettement ou inachèvement 》 (Choix de textes polémiques et théoriques sur la comedia, dans Théâtre espagnol du XVIIe siècle, dir. R. Marrast, Paris, Gallimard, « Bibliothèque de la Pléiade », 1999, t. II, p. 1935-1937). La meilleure voie d'accès à ces textes demeure A. Porqueras Mayo et F. Sánchez Escribano, Preceptiva dramática española del Renacimiento y el Barroco, Madrid, Gredos, 1972. 


\section{Christophe Couderc}

Différentes causes peuvent expliquer ce qui pourrait apparaître aux yeux des critiques modernes comme une insuffisance théorique. Parmi ces explications, la principale a sans doute à voir avec le constat que dans l'Espagne des XVIe et XVII siècles, le théâtre est un art autant qu'une pratique sociale, et que la représentation dramatique est un produit culturel, associé à une industrie du spectacle - industrie plutôt lucrative, selon différents témoignages, et directement associée à une économie de la charité qui a garanti la pérennité de l'activité théâtrale ${ }^{5}$. Le théâtre étant une marchandise, le poète est soumis à la loi de l'offre et de la demande, de sorte que les évolutions de l'esthétique dramatique doivent être comprises en fonction de l'histoire du goût et, donc, de la réception de la comedia par les spectateurs / consommateurs / prescripteurs, plutôt qu'en fonction de débats théoriques intéressant les seuls lettrés. Ce rapport entre pratique et théorie n'a rien d'exclusif à l'Espagne. Ce qui l'est davantage est peut-être la précocité du développement du théâtre moderne, de sorte que lorsque les débats qui avaient agité les milieux lettrés en Italie trouveront des échos en Espagne, la pratique aura déjà imposé ses propres exigences aux dramaturges, en dehors des cadres prévus par les poétiques classicisantes. Dans le débat qui oppose les tenants du classicisme et les apologistes de la comedia nueva, ceux-ci auront donc pour eux l'antériorité d'un théâtre dont la pratique a déjà déterminé les priorités et fixé les conventions.

Le théâtre du Siècle d'or présente enfin la particularité d'avoir rapidement utilisé le seul terme de comedia pour désigner toute œuvre dramatique. Pendant la plus grande partie du XVI ${ }^{\text {e }}$ siècle, l'espagnol disposait d'une riche palette lexicale pour désigner les pièces de théâtre, sans que l'on sache toujours clairement si les appellations variées qui coexistaient (auto, égloga, representación, diálogo, farsa, et quelques autres termes] recouvraient dans la pratique de vraies différences; par ailleurs, à la même époque, les termes de comedia et de tragedia ne désignaient pas spécifiquement des œuvres dramatiques - ce qui pourrait du reste expliquer la multiplication de termes alternatifs visant à dire la spécificité d'une écriture orientée vers la représentation. Malgré cette situation, l'emploi indifférencié du terme comedia pour désigner toute pièce de théâtre va s'imposer à la fin du siècle, générant à moyen et long terme ce que l'on peut appeler avec Francisco Ruiz Ramón un double malentendu, nominaliste et herméneutique, tout se passant comme si l'emploi d'un terme unique avait eu pour conséquence que

5. Pour une évocation plus précise de la vie du théâtre et des conditions matérielles de la représentation, nous nous permettons de renvoyer à C. Couderc, Le Théâtre espagnol du Siècle d'Or (1580-1680), Paris, Puf, 2007, p. 41-55. 
la comedia ne soit pensée que comme un genre hybride, ou plus précisément comme si à ce terme unique ne pouvait correspondre qu'une poétique anti- ou non aristotélicienne, c'est-à-dire qui ne tiendrait compte ni des règles, ni de la division des genres ${ }^{6}$.

Dans ce contexte qui favorise l'hybridité générique, le terme de tragicomedia et ce que ce terme recouvre méritent par conséquent une attention particulière ; l'examen de quelques préfaces, prologues et autres dédicaces rassemblés pour leur édition dans le cadre du projet Les idées du théâtre nous semble pouvoir contribuer utilement à une réflexion portant sur la désignation autant que sur le genre.

\section{La Célestine}

Le cas espagnol présente une particularité en raison de l'importance de la diffusion et, par conséquent, de l'influence de La Célestine. Le titre de cette œuvre hybride ne s'est fixé sous la forme que nous lui connaissons aujourd'hui que dans le courant du XVI ${ }^{\mathrm{e}}$ siècle. Dans son état primitif, la pièce en prose s'intitule Comedia de Calisto y Melibea [Comedia de Calixte et Mélibée], premières éditions en 1499 et 1500), tandis que, dans un second état du texte, modifié par une opération d'allongement et de réécriture, Fernando de Rojas en change le titre en Tragicomedia de Calisto y Melibea? La partie finale du prologue ajouté à cette occasion se propose de justifier la nouvelle étiquette générique. Partant du lieu commun, remontant à Héraclite en passant par Pétrarque, selon lequel il n'y aurait que combat ou bataille (polemos) entre toutes choses, Rojas termine d'illustrer ce principe en évoquant de supposés désaccords sur la question de savoir si l'étiquette générique de «comédie » convient à La Célestine (qui ne s'appelle donc pas encore ainsi] :

6. F. Ruiz Ramón, Calderón nuestro contemporáneo. El escenario imaginario: ensayo sinóptico, Madrid, Castalia, 2000, p. 36. Voir, sur ces questions, C. Couderc, Le théâtre tragique en Espagne au Siècle d'Or. Cristóbal de Virués, Lope de Vega, Calderón, Paris, Puf / Cned, 2012, p. 34-36.

7. Cette complexe histoire du texte est ainsi résumée par Pierre Heugas : «Tels sont les développements de La Célestine par états successifs : $1^{\circ}$ un acte anonyme, $2^{\circ}$ une comédie en seize actes, $3^{\circ}$ une tragi-comédie en vingt et un actes. Si l'on s'en tient aux pièces liminaires et finales, Fernando de Rojas a donc "achevé" la comédie puis l'a reprise, amendée, réécrite dans cet état définitif qu'est la tragi-comédie » (Théâtre espagnol du XVI $l^{e}$ siècle, dir. R. Marrast, Paris, Gallimard, « Bibliothèque de la Pléiade », 1983, p. 848). 


\section{Christophe Couderc}

que dix personnes viennent à se réunir pour entendre cette comédie, en lesquelles il y a tant d'humeurs différentes comme il arrive toujours, niera-t-on qu'il n'y ait motifs de discussion sur des choses qui de tant de façons différentes se peuvent entendre ? [...] Pour d'autres le litige a porté sur le titre et ils ont dit qu'on ne devait point l'appeler comédie, puisqu'elle s'achève en tristesse, mais tragédie. Le premier auteur voulut la désigner d'après le début, qui fut le plaisir, et l'appela comédie. Moi, voyant ces désaccords, j'ai tranché la querelle entre les deux extrêmes et je l'ai appelée tragi-comédie. ${ }^{8}$

Les quelques mots, non dépourvus d'humour, consacrés par Rojas à cette question de la dénomination générique ont-ils eu une influence, et laquelle, sur l'emploi du terme et sur la conception de ce que peut être la tragi-comédie en Espagne ? II est en effet indéniable que l'œuvre a eu un impact considérable. Véritable best-seller ${ }^{9}$, La Célestine a suscité de nombreuses imitations, parfois présentées explicitement comme des suites, dont les titres reprennent parfois - mais pas toujours, loin de là - la désignation de tragi-comédie ${ }^{10}$. Le fait que Pierre Heugas ait utilisé l'expression « la célestinesque » pour rassembler la « descendance » de l'œuvremodèle témoigne de l'embarras des spécialistes devant la question de savoir quelle définition générique il faut donner à La Célestine, et même de savoir si ce texte appartient au genre dramatique, dans la mesure où il ne respecte pas les conventions du théâtre médiéval sans pour autant pouvoir être considéré comme une préfiguration de la formule de la comedia nueva qui ne triomphera sur la scène espagnole qu'un siècle plus tard ${ }^{11}$.

8. « Cuando diez personas se juntaren a oír esta comedia [...] Otros han litigado sobre el nombre, diciendo que no se había de llamar comedia, pues acababa en tristeza, sino que se llamase tragedia. El primer autor quiso darle denominación del principio, que fue placer, y llamola comedia. Yo, viendo estas discordias, entre estos estremos partí agora por medio la porfía y llamela tragicomedia » (F. de Rojas ( $y$ « antiguo autor 》), La Celestina. Tragicomedia de Calisto y Melibea, éd. Francisco J. Lobera et al., Barcelone, Crítica, 2000, p. 20-21).

9. Son éditeur et traducteur Pierre Heugas n’hésite pas à qualifier La Célestine de «best-seller 》 (Théâtre espagnol du XVle siècle, éd. cit., p. 849).

10. C'est le cas de la Tragicomedia de Lisandro y Roselia, de Sancho de Muñón. Mais d'autres textes, parmi les plus connus de ces suites, sont intitulés « comédies 》 (la Comedia Floriena de Juan Rodríguez del Padrón, la Comedia Selvagia d’Alonso de Villegas), ou bien « tragédies » (Sebastián Fernández, Tragedia Policiana).

11. La Célestine pourrait avoir été conçue comme le support d'une lecture dramatique, comme le laisse entendre le prologue précédemment cité [« que dix personnes viennent à se réunir pour entendre cette comédie ») ainsi qu'un autre passage du paratexte situé à la fin de l'œuvre : « Si tu aimes, si tu veux, en lisant l'histoire de Calixte / Amener tes auditeurs à beaucoup d'attention / II faut que tu saches parler entre les dents, / Tantôt avec colère et grand désespoir. / Prends en lisant mille façons et manières. / Questionne et réponds par la bouche de tous, / Pleure et ris au 
Telle que la comprend Rojas, la notion de tragi-comédie recouvre un sens assez étroit - ne serait-ce que parce que la formulation qui la contient est très brève. Littéralement, le terme semble en effet approprié à l'auteur en fonction du seul critère de la nature du dénouement (heureux ou malheureux), et il faut avoir recours à d'autres éléments présents dans le paratexte pour contextualiser cette succincte mention de la tragi-comédie et la compléter. La critique s'y est légitimement employée, sur deux points. En premier lieu, la conception proprement dramatique de La Célestine étant affirmée par ailleurs dans le paratexte (notamment moyennant une référence à Térence ${ }^{12}$ ], il est loisible de considérer que cet emploi du terme de tragi-comédie fait écho-mais implicitement - au prologue de l'Amphitryon ${ }^{13}$, où apparaît pour la première fois le terme de tragicocomœdia, créé par Plaute ${ }^{14}$ pour désigner une action risible (du ressort de la comédie) dont les personnages sont de naissance illustre (du ressort de la tragédie] - et l'on sait que la référence deviendra un lieu commun dans les débats autour de la tragi-comédie ${ }^{15}$. Relevons au passage que la variante du mélange des genres illustrée par La Célestine propose, à l'inverse, une action comique (du fait de son sujet et de la naissance moyenne des personnages) terminée par un dénouement tragique (mort des personnages principaux). Le second point sur lequel il est fondé de compléter la brève référence à la tragi-comédie contenue dans le prologue concerne la complexe question de l'exemplarité d'une fiction dont

bon moment. 》 («Si amas y quieres a mucha atención / leyendo a Calisto mover los oyentes, / cumple que sepas hablar entre dientes : / a veces con gozo, esperanza y pasión, / a veces airado, con gran turbación. / Finge, leyendo mil artes y modos; / pregunta y responde por boca de todos, / llorando y riendo en tiempo y sazón »] [La Celestina, éd. cit., p. 352-353).

12. Sur ce point, voir les arguments développés par N. Salvador Miguel, « La Celestina », dans J. Huerta Calvo (dir.), Historia del teatro español, Madrid, Gredos, 2003, t. I, p. 137-16? (notamment p. 150).

13. Certains éditeurs préfèrent se montrer prudents et sur ce point ne pas écarter l'éventualité d'une polygénèse : « C'est probablement une imitation du mot latin tragico[co]moedia, qui apparaît seulement, avant La Célestine, dans le prologue de l'Amphitruo de Plaute et dans celui de Fernandus servatus de Carlo y Marcellino Verardi (1493), même s'il est également possible que Rojas ait inventé ce terme de façon indépendante » (J. Lobera et al., dans La Celestina, éd. cit., p. 21, n. 51 ; nous traduisons].

14. Voir C. Auvray-Assayas, « Le mélange des genres ou comment les Romains pensent leur identité littéraire », dans M. Torres, A. Ferry, S. Moncó Taracena et D. Lecler (éd.), Tragique et comique liés, dans le théâtre, de l'Antiquité à nos jours (du texte à la mise en scène), Rouen, Publications numériques du CÉRÉdl, n ?, 2012 (http://ceredi.labos.univ-rouen.fr/public/?lemelange-des-genres-ou-comment.html).

15. Une recherche par mot clé dans la base de données du site ldT est sur ce point très instructive. 


\section{Christophe Couderc}

le caractère passablement scandaleux pourrait avoir amené l'auteur à la légitimer du mieux possible en tirant son œuvre vers le genre de la tragédie, plus sérieux et plus respectable que la comédie ${ }^{16}$. La reprobatio amoris comme justification d'une action scabreuse est en effet cohérente avec les propos d'un auteur qui dit préférer le terme de tragi-comédie à celui de comédie juste avant d'expliquer qu'il a réalisé une dernière opération d'amplification du texte initial afin de satisfaire la demande de ses premiers lecteurs de «prolonger le cours du plaisir des amants ${ }^{17}$ »; or le 《plaisir 》 [《deleite 》) est le terme utilisé précédemment par Rojas pour justifier que l'auteur de la version primitive ait appelé son œuvre « comédie » : «e premier auteur voulut la désigner d'après le début, qui fut le plaisir, et l'appela comédie ».

De ces deux observations (l'étiquette mixte de tragi-comédie et la revendication de la moralité de l'œuvre) a ainsi pu découler une lecture associant assez étroitement, dans la tradition espagnole, le concept de tragi-comédie avec celui d'une action dramatique dont le dénouement est funeste, parce que par là même il est exemplaire, alors que son action présente des traits caractéristiques de la comédie ${ }^{18}$. La tragi-comédie, selon Rojas, désigne ainsi un mélange qui n'est pas n'importe quel mélange : l'auteur ne parle pas explicitement du critère de la condition sociale des personnages pour différencier la tragédie et la comédie, mais considère que la tragicomedia finit mal, ce qui implique que le dénouement est un critère fondamental de sa définition. Alors que la comédie commence mal et finit bien, la tragédie commence bien et finit mal, et l'histoire de Calixte et Mélibée, nous dit Rojas, est d'un genre hybride parce que, sans présenter pour autant tous les signes distinctifs du genre tragique, elle finit par la mort des amants alors qu'elle débutait dans la joie. On retiendra des remarques qui précèdent que la tragicomédie est conçue, en fonction de ce modèle, comme une action foncièrement

16. Nous renvoyons aux judicieuses remarques de $P$. Heugas sur ce point complexe qu'il appelait « le problème, capital pour nos œuvres, de la légitimité du docere delectando [enfermé] dans une alternative : la référence au docere delectando est-elle un lieu commun linguistique ou stylistique constant, ou bien traduit-elle un aspect doctrinal également constant dans le prologue du XVI ${ }^{e}$ siècle ? » (P. Heugas, La Célestine et sa descendance directe, Bordeaux, Institut d'Études Ibériques et Ibéroaméricaines, 1973, p. 546].

17. « alargar [...] el proceso de su deleite destos amantes » (La Celestina, éd. cit., p. 21 ; dans Théâtre espagnol du XVI siècle, éd. cit., p. 848].

18. Ainsi, lorsque S. Fernández, dans sa Tragedia policiana (1547), imite La Célestine, il qualifie bien son œuvre de tragédie, et la fait précéder d'un prologue dans lequel il justifie la peinture des amours des héros par la finalité morale, de sorte que «sous une apparence ridicule les jeunes gens soient mis en garde contre les désastres que l'amour dissimule sous l'attrait du plaisir mondain » (« debajo de algún color ridículo tomen aviso los vanos mançebos de los desastres que el amor encubre con el çevo del deleyte mundano », cité par M. Newels, op. cit., p. 128, n. 10). 
comique mais qui finit mal, sans que rien ne soit dit d'une compréhension du concept, ou de son application, ni au cas d'une tragédie à fin heureuse, ni - du moins explicitement - au cas d'une action mêlant des personnages de statut social différent.

C'est pourtant suivant ces deux acceptions que le terme va être utilisé en Espagne dans les décennies suivantes, et encore bien plus tard, lorsqu'il s'agira de défendre la comedia nueva, celle dont Lope de Vega contribuera fortement à fixer la formule. Autrement dit, si le succès de La Célestine n'est sans doute pas étranger au fait que l'on a défendu consciemment, et très tôt en Espagne, une pratique de l'hybridité générique, il convient de prendre en compte que le terme de tragicomedia recouvre tôt des combinaisons différentes du tragique et du comique.

II est certain que les exemples ne manquent pas de mélange du tragique et du comique dans les textes de théâtre publiés en Espagne au XVI ${ }^{e}$ siècle, parfois précédés d'un paratexte attestant que la pratique de l'hybridité générique était consciente. C'est le cas de la Comedia Thebayda, texte anonyme publié en $1521^{19}$. Son paratexte (《prefactión » en prose puis « epigrama » adressés au duc de Gandía) doit être placé dans le droit fil de celui de La Célestine, notamment pour la volonté déclarée de proposer une fiction qui puisse servir d'avertissement sur les périls de l'amour, mêlant par conséquent la peinture des actions lascives (« actos venéreos ») et les sentences (《sentencias 》). Si la variété des matériaux et des tonalités est justifiée de la même façon, à savoir par l'utilité morale (avec une référence explicite à l'utile dulci d'Horace), l'alliage de comédie et de tragédie, cependant, n'est pas celui que I'on trouve dans La Célestine. En effet, dans la Comedia Thebayda, comme le souligne José Luis Canet quand il commente les vers où le dramaturge anonyme s'attarde à énoncer « la manière et le style de son $œ u v r e^{20} 》$, « l'auteur est conscient de ce que la matière amoureuse est tragique (comme c'est le cas dans toute la fiction sentimentale et dans de nombreuses églogues de cour] mais, ne pouvant enfreindre la stricte règle des styles, il est

19. Anonyme, Comedia Thebayda, (1521), Prefactión ; éd. J. L. Canet, dans site IdT - Les idées du théâtre (http://www.idt.paris-sorbonne.fr), n. 19.

20. « [L'auteur] déclare la manière et le style de l'œuvre : suivant des matières qui sèment l'épouvante / et fort remarquables dans cette comédie / de telle sorte que j'imite la tragédie / mais non en son début ni en sa fin » [ «Declara la manera y estilo de la obra : Siguiendo materias que ponen espanto / y bien peregrinas en esta comedia, / y tanto que imito sin duda a tragedia / mas no en los principios y fin [le] quebranto »] (ibid.; nous traduisons ). 


\section{Christophe Couderc}

contraint de modifier le début et la fin de l'histoire, qui commence dans la tristesse et finit dans le bonheur ${ }^{21} \gg$.

Quel qu'ait été l'impact réel du best-seller que fut La Célestine, il est indéniable, comme ont pu le relever Herrick ou Newels, que le terme de tragicomedia a été employé avec une fréquence relativement élevée après sa publication ${ }^{22}$. Plus encore, Margarete Newels a rassemblé un certain nombre d'exemples d'une pratique, parfois consciente, du mélange des genres que la tradition scolastique et la poétique néo-aristotélicienne tenaient pour incompatibles. Ainsi Juan de la Cueva ouvre-t-il son Romancero, publié en 1587 à Séville, par un texte adressé « Au lecteur » dans lequel le poète indique que la pratique mêle désormais le tragique et le comique, Euripide et Térence, Plaute et Sénèque ${ }^{23}$. La même spécialiste va jusqu'à considérer avec García Soriano que le théâtre universitaire pratiquait lui aussi une forme mixte que l'on peut déjà assimiler à une véritable tragi-comédie ${ }^{24}$. Oleza peut, par conséquent, différencier deux acceptions de la tragi-comédie qu'il propose d'ordonner diachroniquement : tandis que le modèle de La Célestine proposait une tragédie assortie d'épisodes ou de personnages comiques, l'autre variété de tragi-comédie, très pratiquée par les dramaturges espagnols au XVIe siècle (par exemple Torres Naharro), est une comédie qui joue avec un risque tragique pour finalement l'éviter ; Lope de Vega et ses contemporains, selon le

\section{Ibid., n. 19. Nous traduisons.}

22. M. T. Herrick, Tragi-comedy, its origin and development in Italy, France, and England, Urbana, University of Illinois Press, 1955 ; M. Newels, op. cit.

23. «Quoique désormais la tragédie et la comédie / soient une seule et même chose, / puisque dans toutes deux on représente / des hommes de haute et de basse naissance, / de sorte que ni par les costumes, ni par les personnages, / en rien elles ne se différencient, / et que tout est désormais / une même toile et une même livrée, / Euripide et Térence, / le gai Plaute et Sénèque 》 [« Aunque el Trágico, y el Cómico, / es uno ya, y una cuenta, / que hombres altos, y hombres bajos, / en ambos se representa, / qu'en vestido, ni en personas, / en nada se diferencian, / assí, que ya es todo uno, / un paño, y una librea, / Eurípides, y Terencio, / El risueño Plauto y Séneca »] [ Juan de la Cueva, «Al lector », dans Coro febeo de romances historiales, cité par Newels, op. cit., p. 137 ; nous traduisons).

24. «Les comédies de collège, dans leur expression la plus générale et la plus caractéristique, furent de véritables tragi-comédies qui annonçaient la naissante immédiatement postérieure du théâtre espagnol et l'avènement ultérieur du drame romantique » [《Fueron, pues, las comedias de colegio, en su manifestación más general y característica, verdaderas tragicomedias que anunciaban el nacimiento inmediato del teatro español y el ulterior arribo del drama romántico »] [J. García Soriano, Teatro universitario y humanístico, cité par M. Newels, op. cit., p. 129 ; nous traduisons ). 
même critique, retrouveraient en quelque sorte la première combinaison en faisant pencher le fléau de la balance tragi-comique vers la tragédie ${ }^{25}$.

\section{La nouvelle tragicomedia}

Le fait est qu'avec l'avènement de la nouvelle esthétique dramatique, c'està-dire de la formule qui s'impose à mesure que se développe l'activité des corrales de comedias dans les villes les plus importantes d'Espagne, la réflexion autour de la tragicomedia peut sembler resurgir avec une acuité particulière, la question se posant dès cette époque de savoir si tout le théâtre qu'on joue alors dans le cadre de cette activité mercantile peut être désigné grâce à cette étiquette. Le terme de tragi-comédie va dès lors se retrouver au carrefour des débats sur la question du genre du théâtre de cette époque, mais il va également pouvoir être instrumentalisé, peut-être simplement en raison de la nature nettement polémique de ces mêmes débats, mais également parce que, dans le cas de Lope de Vega, des enjeux personnels sont étroitement liés à la défense de la dignité littéraire de la comedia nueva.

Dans le contexte des polémiques qui accompagnent l'affirmation puis le triomphe définitif de la comedia nueva ${ }^{26}$, Ricardo de Turia - pseudonyme d'un auteur dont l'identité n'est pas encore tout à fait certaine - affirme ainsi dans son Apologético de las comedias españolas [Apologie en faveur des comédies d'Espagne], qui fait partie des textes liminaires de l'anthologie Norte de la poesía española [Nord de la poésie espagnole] publiée à Valence en 1616 : « aucune des "comédies" parmi toutes celles représentées en Espagne ne mérite ce nom, mais bien celui de tragi-comédie ${ }^{27} \gg$. Turia est très ouvertement polémique, comme le laisse voir l'entrée en matière, mordante et spirituelle, qui lui permet de s'en

25. Voir J. Oleza, From Ancient Classical to Modern Classical : Lope de Vega and the New Challenges of spanish Theatre, New York, Idea, 2012, en particulier p. 33-36 : « The Spanish Comedy was essentially comedy with shades of tragedy during the whole Renaissance period, to retake with Tárrega and Lope the gesture of Rojas, the tragedy with comedy laughters » (p. 35).

26. Sur la double controverse, éthique et esthétique, qui accompagne le triomphe du nouveau théâtre en Espagne, voir M. Vitse, Éléments pour une théorie du théâtre espagnol du XVII siècle, Toulouse, Presses Universitaires du Mirail, 1990, ainsi que M. Vitse, « Le premier essor du théâtre », dans J. Canavaggio (dir.), Histoire de la littérature espagnole, Paris, Fayard, 1993, t. 1, notamment p. 603-606.

27. R. de Turia, Apologético de las comedias españolas (1616) ; éd. A. García-Reidy, site IdT, f. A4rº. Nos références au texte français reprennent la traduction de M. Vitse dans Théâtre espagnol du XVII siècle, éd. cit., p. 1425-1429. 


\section{Christophe Couderc}

prendre aux adversaires du nouveau théâtre, qu'il appelle les « sectateurs de Térence et de Plaute 》 ( « teresiarcos 》 et «plautistas 》 dans le texte original ${ }^{28}$ ) : à ces défenseurs des règles, il prête une vision hypercritique de la comedia nueva, pour habilement revendiquer comme des qualités ce que les adversaires du nouveau théâtre jugent comme des défauts. Comme Alejandro García-Reidy l'a relevé dans son édition de l'Apologie ${ }^{29}$, malgré l'intérêt de ce texte, Turia est peu original dans le choix de ses arguments. Il utilise notamment à plusieurs reprises des formules qui sont un rappel direct de l'Arte nuevo de hacer comedias en este tiempo que Lope de Vega avait publié en 1609 (en appendice à ses Rimas) ou encore du prologue aux lecteurs signé par Gaspar de Porres (mais probablement écrit par l'auteur) qui ouvrait la Parte IV des comedias de Lope de Vega (volume publié en 1614] : I'utilisation du terme même de tragicomedia peut être considérée comme une explicitation de ce qui demeure implicite dans les vers de l'Arte nuevo, devenus célèbres, où Lope semble bien déclarer que tout le théâtre joué à présent en Espagne, semblable à un monstre, mêle des caractéristiques propres à la comédie et à la tragédie :

Le tragique au comique une fois mélangé, Et Térence et Sénèque - en dût-il résulter Quelque autre Minotaure de Pasiphaé-, Permettra d'alterner le grave et le plaisant : Cette diversité est pleine d'agrément. ${ }^{30}$

La mention de «l'humeur espagnole » (« cólera española » dans le texte de Turia ${ }^{31}$ ] ne peut que rappeler la même formule employée précédemment par Lope

28. Sur les deux termes de terensiarcos et plautistas, voir R. de Turia, Apologético (1616), éd. cit., n. 1.

29. Ibid.

30. « Lo trágico y lo cómico mezclado, / y Terencio y Séneca, aunque sea / como otro Minotauro de Pasife, / harán grave una parte, otra ridícula, / que aquesta variedad deleita mucho » (Lope de Vega, Nouvel art de faire des comédies en ce temps, v. 174-178, trad. A. Labertit, dans Théâtre espagnol du XVII ${ }^{e}$ siècle, éd. cit., p. 1419].

31. « L'humeur espagnole se satisfait davantage de la peinture que de l'histoire[.] Voilà pourquoi les Espagnols, emportés par leur naturel, voudraient voir en une seule "comédie" non seulement la prodigieuse naissance d'un prince, mais encore les exploits que promettent d'aussi extraordinaires commencements [...]. Et, pareillement, dans ce court espace de deux heures, ils voudraient voir des péripéties. 》 (Apologie, éd. citée, p. 1427-1428). Cf. « Tout de bon, si l'on considère que l'humeur / D'un Espagnol assis ne peut se tempérer / $\mathrm{Si}$, durant deux heures de temps, il n'a tout vu / Filer de la Genèse au Jugement dernier » (Lope de Vega, Nouvel art de faire des comédies en ce temps, éd. cit., p. 1420). 
dans les vers 205-210 de son épître, et sert pareillement à Turia à justifier un manquement à l'unité de temps ; le fait de considérer comme « premier précepte d'Aristote dans sa Poétique »la recherche des « applaudissements » rappelle directement l'argumentation de Lope $^{32}$; l'association de la «pure » comédie avec l'entremés (brève pièce, proche de la farce, que l'on représentait en Espagne entre deux actes de la comedia) fait également partie de la stratégie adoptée par Lope dans l'Arte nuevo afin de donner plus de dignité littéraire à la comedia en la différenciant du genre comique ${ }^{33}$; la référence récurrente au «laquais » (le gracioso], symbole de la grossièreté du genre comique, est aussi fréquente chez Lope, qui entend à toute force s'en démarquer ${ }^{34}$, ce qui permet de supposer que la

32. Cf. « bien peu nombreux doivent désormais être les observateurs scrupuleux de notre théâtre à qui il ne semble évident que satisfaire le vulgaire est le seul précepte et la seule loi que l'on suive en Espagne quand on écrit pour le théâtre ; c'est une maxime qui était déjà du goût d'Aristote quand il disait que l'auteur de la pièce touchait son but s'il procurait du plaisir à ses auditeurs »: [ « ya pocos deben ser los escrupulosos a quien no conste que no hay en España más preceptos ni leyes para las comedias que satisfacer al vulgo, máxima que no desagradó a Aristóteles cuando dijo que el poeta de la fábula había conseguido el fin con ella si conseguía el gusto de los oyentes »] (Lope de Vega, Parte IV [1614), « A los lectores »; éd. L. Giuliani, site IdT, NP1; nous traduisons]. Ce dernier texte, s'il est bien de Lope (ce qui est très probable), a permis à celui-ci de contribuer à faire de l'Arte nuevo un texte de référence dans les débats postérieurs autour de la Comedia nueva. La phrase qui précède cette citation fait en effet allusion à l'épître publiée cinq ans plus tôt : « Je renvoie les fâcheux au texte qu'il écrivit pour sa défense devant l'Académie de Madrid et qui a été imprimé à la suite de ses Rimes 》 [ « Remito a los quejosos al que él escribió para su defensa en la Academia de Madrid, que anda impreso en sus Rimas »; nous traduisons].

33. La proposition « ce qui est purement comique fournissant la matière des intermèdes tels qu'on les conçoit aujourd'hui » (Turia, Apologie, op. cit., p. 1428) rappelle celle de Lope «l'habitude est restée / De dénommer intermèdes ces comédies / Anciennes où l'art paraît dans toute sa force, / Où l'action est unique et entre gens du peuple » (Lope de Vega, Nouvel art de faire des comédies en ce temps, éd. cit., p. 1416-1417).

34. Dans une lettre souvent citée datant de la fin 1630, Lope se plaint au duc de Sessa de l'insuccès qu'ont rencontré ses dernières pièces et se réfère à ce même «laquais de comédie 》 comme symbole d'une activité indigne (notamment pour le prêtre qu'il est devenu quinze ans auparavant ] : « après que deux de mes pièces, bien écrites pourtant mais mal reçues, ont déplu au vulgaire, j'ai compris que soit on veut de jeunes pousses, soit le Ciel ne veut pas que la Mort trouve un prêtre en train d'écrire les répliques d'un laquais de comédie ; aussi me suis-je proposé de les abandonner définitivement, ne voulant pas finir comme les jolies femmes, dont tout le monde se moque quand elles sont devenues vieilles 》 [ « con desagradar al pueblo dos historias que le di bien escritas y mal escuchadas, he conocido, o que quieren verdes años, o que no quiere el cielo que halle la muerte a un sacerdote escribiendo lacayos de comedias, he propuesto dejarlas de todo punto, por no ser como las mujeres hermosas, que a la vejez todos se burlan de ellas »] (Epistolario de Lope de Vega Carpio, éd. A. González de Amezúa, Madrid, Real Academia Española, t. IV, p. 143-144; nous modernisons l'orthographe et traduisons]. 


\section{Christophe Couderc}

critique du mauvais goût, c'est-à-dire du caractère populaire du nouveau théâtre, faisait partie des arguments récurrents qui, dans la polémique autour de la comedia, nuisaient probablement - y compris sur le plan personnel - directement au «Phénix des Beaux Esprits »; ou encore l'idée selon laquelle le refus des règles, loin d'être une preuve de leur ignorance, est le fruit d'un choix pour les auteurs espagnols, qui sont du reste parfaitement capables (comme Lope l'affirme, là encore dans l'Arte nuevo] d'écrire en suivant les préceptes classiques:

Et l'on aurait bien tort de penser que la non-observation de ces règles vient du fait qu'ils en ignorent l'existence. Sans compter que les auteurs de «comédies 》 de notre époque, ayant par ailleurs fait leurs preuves avec d'autres œuvres parfaitement achevées et qu'ils ont écrites avec une tout autre finalité que celle de satisfaire la multitude, n'ont guère besoin, pour rendre leurs noms immortels, d'écrire leurs « comédies » avec la rigueur à quoi veulent les contraindre ces censeurs guindés auxquels s'adresse mon Apologie. ${ }^{35}$

De façon générale, Turia suit parfaitement Lope dans la revendication de la modernité de la comedia nueva, dans l'affirmation de la supériorité du théâtre espagnol sur tous les autres, étrangers ou antérieurs, dans la conscience de la nécessaire adéquation du théâtre aux temps nouveaux : nous sommes bien là, comme l'a indiqué Vitse, face à une variante de la querelle entre Anciens et Modernes, et face à l'une des expressions de la modernité anticlassique, pour reprendre une formulation de Georges Forestier ${ }^{36}$. Quant aux arguments un peu plus précis que Turia avance pour justifier que toutes les pièces jouées en Espagne puissent être considérées comme des tragi-comédies, ils sont aussi dans l'air du temps. Qu'il s'agisse de définir la tragi-comédie comme un poème «mixte » (et non pas composé, suivant une distinction aristotélicienne), dans lequel « les éléments perdent leur forme et constituent une tierce matière très différente », ou de la comparer au « fabuleux Hermaphrodite [...] qui, né d'un homme et d'une femme, était de nature différente de ces deux sexes, mêlés de telle manière que l'on ne

35. Turia, Apologie, éd. cit., p. 1427.

36. M. Vitse, « Le premier essor », art. cit., p. 603. G. Forestier désigne de la sorte le mouvement européen de rénovation théâtrale dans lequel les dramaturges sont invités à s'éloigner des règles antiques ( «De la modernité anticlassique au classicisme moderne. Le modèle théâtral [1628-1634] 》, Littératures Classiques, n 19, automne 1993, p. 87-127 ; repris dans Passions tragiques et règles classiques. Essai sur la tragédie classique française, Paris, Puf, 2003, p. 29-70). Voir sur ce point les remarques de Florence d'Artois dans « Pourquoi n'y a-t-il pas eu de tragédie classique en Espagne ? », Europe, n¹002, octobre 2012, p. 56-?0. 
pouvait les séparer l'un de l'autre », Turia n'est guère novateur ${ }^{37}$. Pas plus originale - mais tout aussi révélatrice de la dimension européenne des débats portant sur la tragi-comédie - est la recherche de précédents au principe du mélange, que Turia ne trouve pas seulement dans la tragi-comédie mais dans les deux genres, qu'il appelle explicitement «purs », que sont la tragédie et la comédie :

Lors donc, si dans un poème dit «pur», comme la tragédie et la comédie, on observe cette coexistence de personnes illustres et de celles qui ne le sont pas, pourquoi s'étonner de la retrouver dans ce poème mixte qu'est la tragicomédie ? Outre que les Espagnols n'ont pas été les inventeurs de ce poème mixte (l'auraient-ils été que leur renommée n'en fût pas amoindrie) : il est fort ancien et, dans tous les cas, a permis au talent du poète de briller plus encore en raison de l'extrême artifice qu'implique, en soi, le mélange de choses si distinctes et si variées. ${ }^{38}$

On trouve là un exemple, extrêmement courant par ailleurs, de justification de l'irrégularité et du mélange par des exemples pris chez les anciens, de même que dans d'autres cas l'on renverra à l'autorité d'Aristote. En amont de l'Arte nuevo de Lope, il est probablement nécessaire de référer le texte de Ricardo de Turia à la Filosofía antigua poética, sorte de longue glose dialoguée de la Poétique rédigée par Alonso López Pinciano entre 1588 et 1593, publiée en 1596, et probablement lue par de nombreux écrivains (dont Lope de Vega). Ainsi l'image de l'hermaphrodite utilisée par Turia n'est-elle pas sans rappeler, en plus du Minotaure des célèbres vers de l'Arte nuevo cités plus haut, celle du monstre, évoquée au chapitre VII de la Poétique d'Aristote et dans les premiers vers de l'Art poétique de Horace, que reprend López Pinciano dans sa Filosofía antigua poética afin de justifier l'existence de la tragi-comédie. Pour ce faire, López Pinciano fait en effet référence à Plaute, en mettant principalement en avant le critère de la naissance des personnages. Lope de Vega, après lui et à notre avis à son exemple ${ }^{39}$, en fera autant dans l'Arte nuevo : c'est du moins ce que pourrait indiquer l'utilisation

37. Comme le résume A. García-Reidy dans sa Présentation du texte de R. de Turia pour le site $I d T$ : «l'auteur se base sur le Compendio della poesia tragicomica de Gian Battista Guarini, publié pour la première fois en 1601, qui accompagne le texte de /l pastor Fido depuis l'édition de 1602. Des passages tels que la différence entre le mélange-fusion et le mélange-agrégation établie par Aristote, la référence au légendaire Hermaphrodite, la caractérisation de la tragédie et de la comédie ou la mention de Sophocle et Aristophane comme exemples de dramaturges de l'Antiquité qui mêlèrent dans leurs œuvres différents éléments, tout cela provient directement du texte de Guarini » (R. de Turia, Apologético, éd. cit.).

38. R. de Turia, Apologie, éd. cit., p. 1428.

39. Voir aussi M. Newels, op. cit., p. 143. 


\section{Christophe Couderc}

par les deux auteurs de la même paire d'adjectifs (《 sérieux 》 et « ridicule », «grave » et «ridículo 》) pour qualifier en les opposant les personnages propres à la tragédie et à la comédie ${ }^{40}$. Si l'Amphitryon de Plaute peut donc être qualifié de tragi-comédie, López Pinciano considère qu'il en va de même de l'Odyssée - l'exemple pris par Aristote aux chapitres XIII et XVIII de la Poétique d'une action complexe fondée sur le caractère, ou les mœurs - du fait de la naissance de ses personnages principaux, engagés cependant dans une action causant chez le spectateur les émotions qui sont celles de la comédie. Constatant sur ce point que « certains poètes de notre époque qualifient de monstres ces mélanges, quoique je leur aie dit, mais en vain, que Plaute a appelé tragi-comédie son $A_{m p h i t r y o n}{ }^{41}{ }$, López Pinciano se réfère ensuite à des exemples de tels alliages, qu'il justifie en les référant aux pratiques anciennes et à l'autorité d'Aristote. Même s'il préfère les formes pures, il renvoie à Aristote pour dire, en utilisant à nouveau le terme de «monstre », que ce mélange est possible et licite (« se pueden mezclar estas especies sin hacer monstruos, sino criaturas muy bellas »).

Les textes théoriques, et parmi eux les paratextes, peuvent de la sorte être disposés en un réseau supposant des renvois de l'un à l'autre, des réponses plus ou moins polémiques, qui ne permettent le plus souvent pas autre chose que des hypothèses. Ainsi, les termes de monstre et d'hermaphrodite sont présents chez Turia et aussi chez Francisco Cascales. Celui-ci publie en 1617 (soit un an à peine après le texte de Ricardo de Turia - mais il n'est pas impossible que le texte de Cascales soit antérieur] ses Tablas poéticas où il constate - pour s'en attrister que l'on ne représente pas en Espagne de véritables tragédies mais, fait-il dire aux deux personnages qu'il fait dialoguer, des pièces monstrueuses : elles ont tout de la tragédie, et si leur fin n'est pas funeste, cela ne suffit cependant pas à les faire considérer comme des comédies, car leur « matière 》 (ou, plus loin, « le corps de la fable »), c'est-à-dire leur sujet [《sujeto »), n'est pas celui de la comédie.

40. « L'Amphitryon de Plaute dont vous parlez n'est pas une pure comédie, puisque Mercure lui-même, dans le prologue, l'appelle tragi-comédie en raison du mélange qui y est fait entre les personnages graves et les ridicules » [ « El Amphitryón de Plauto que decís, no es pura comedia, porque el mismo Mercurio, prologando, la dice tragicomedia por la mezcla que tiene de las personas graves y de lo ridículo »] (A. López Pinciano, Filosofía antigua poética, éd. de A. Carballo Picazo, Madrid, CSIC, 1973, III, p. 21 ; nous traduisons]. Dans le texte original de l'Arte nuevo, « le grave et le plaisant » (dans la citation de la note 30 ) correspond aux mêmes adjectifs : « grave una parte, otra ridícula ».

41. «Algunos poetas de nuestros tiempos dicen que son monstruos estas mezclas, y aunque les he dicho que Plauto llamó a su Amphitryón tragicomedia, no aprovecha » (ibid., p. 219-220; nous traduisons]. 
C'est là une définition assez précise de ce que recouvre à cette époque le terme de tragicomedia, que d'aucuns voudraient utiliser mais que Cascales s'emploie à récuser :

CAStalio. - Ce ne sont pas des comédies, avec lesquelles elles sont sans aucun rapport. Ce sont des hermaphrodites, des monstres de la poésie. Aucune de ces histoires n'a de matière comique, quand bien même elle terminerait dans l'allégresse.

Pierio. - Au moins peut-on les appeler tragi-comédies.

CASTALIO. - Allons bon! Ne vous ai-je pas dit il y a peu le défaut des tragédies et des comédies doubles?

PIERIO. - Pourra-t-on parler de comédies doubles?

Castalio. - Pas le moins du monde, car la comédie double est celle-là où interviennent des princes et de grands personnages, aux côtés de gens du commun, mais elle doit avoir un sujet comique et ses péripéties doivent éveiller le rire et procurer du divertissement.

Pierio. - Appelons-les donc tragédies doubles, puisque tout en elles est du ressort du tragique quoiqu'elles terminent heureusement.

CASTALIO. - Vous vous faites si pressant que je ne peux vous contester cela. Soit, ce sont des tragédies doubles, ce qui revient à dire de mauvaises tragédies; et je leur donne cette appellation de mauvaise grâce, car leur sujet manque fort de ce tragique avec quoi l'on éveille la terreur et la pitié. ${ }^{42}$

Comme López Pinciano dans le texte précédemment cité, et comme s'il l'explicitait, Cascales s'appuie sur Aristote et précise les possibilités de mélange du comique et du tragique : l'objet de sa critique n'est pas tant un type d'action comique dont les sujets seraient de grands personnages (ce qu'il appelle la comédie double, dont l'Amphitryon de Plaute pourrait être l'illustration] que la forme « mixte » consistant

42. « CASTALIO. - No son comedias, ni sombra de ellas. Son unos hermafroditos, unos monstruos de la poesía. Ninguna de esas fábulas tiene materia cómica, aunque más acabe en alegría. Pierio. - A lo menos llamarse han tragicomedias. CASTALIO. - Quita allá. ¿No os he dicho poco ha el vicio de las tragedias dobles y comedias dobles? Pierio. - ¿A lo menos serán comedias dobles? Castalio. - Ni por pensamiento, porque la comedia doble es aquella que lleva algunos príncipes y personas ilustres, juntamente con las humildes, pero ha de tener sujeto cómico y acontecimientos de donde se pueda sacar la risa y pasatiempo. PIERIO. - Llamémoslas, pues, tragedias dobles, ya que el cuerpo de toda la fábula es trágico y para en felicidad. CASTALIO. - Apretáisme de manera que no os puedo negar eso. En fin, son tragedias dobles, que es como decir malas tragedias; $y$ aun este nombre les doy de mala gana, porque tienen muy poco de sujeto trágico con que se ha de mover a misericordia y miedo (F. Cascales, Tablas poéticas, dans F. Sánchez Escribano et A. Porqueras Mayo, op. cit., p. 196-197; nous traduisons]. 


\section{Christophe Couderc}

à donner une fin heureuse à une action de tragédie. II se dit ainsi surtout chagriné par ces pièces qui répondent à la définition de la tragédie à structure double dans la Poétique et qu'Aristote décrit de la façon suivante :

Nous ne mettrons qu'au second rang la structure que certains mettent au premier : la tragédie qui a une structure double, comme l'Odyssée, et qui finit de façon opposée pour les bons et pour les méchants; sa supériorité n'est qu'une apparence due à l'inconsistance du public : car les poètes se laissent mener et se conforment, en composant, aux souhaits des spectateurs. Mais ce n'est pas là le plaisir que doit donner la tragédie, c'est plutôt le plaisir propre de la comédie. ${ }^{43}$

Quoique Cascales soit plutôt tenu pour un « classique » qui admire Horace et pour un adversaire de Lope de Vega, il est incontestable qu'il affine la définition de la tragi-comédie en indiquant qu'elle n'est pas incompatible avec la Poétique, mais, plus encore, qu'elle est en réalité une sous-espèce de la tragédie; une forme de tragédie, plus aimable, moins parfaitement tragique d'après Aristote, mais une tragédie quand même. II est frappant de constater que les termes du débat sont au fond les mêmes que ceux utilisés en France une vingtaine d'années plus tard, quand adversaires et partisans de la tragi-comédie, avec des objectifs opposés, tenteront de dissoudre la tragi-comédie dans la tragédie, soit pour lui donner un lustre et une dignité nécessaires à sa défense, soit pour conclure - d'Aubignac et Sarasin en France, comme Cascales en Espagne - à l'inutilité d'un nouveau terme pour une réalité qui n'a rien de nouveau ${ }^{44}$.

La position de Cascales est donc très claire : derrière l'invective, il expose comment la comedia nueva peut être comprise grâce à la Poétique d'Aristote, et le prix de son témoignage - même s'il ne s'agit pas d'un paratexte - est qu'il ne se contente pas de se placer sur le terrain théorique, mais fait référence à la pratique dont il est témoin. Plus précisément, Cascales récuse doublement la tragicomedia, c'est-à-dire qu'il récuse le concept de tragicomedia, parce que c'est celui d'une forme dramatique imparfaitement tragique selon Aristote, qui dit effectivement que la forme la meilleure de tragédie suppose une action unique conduite de façon rectiligne jusqu'à une issue funeste ; et il récuse également le mot tragicomedia, parce qu'il est inutile de remplacer par ce nouveau terme celui, aristotélicien, de tragédie double.

43. Aristote, Poétique, éd. R. Dupont-Roc et J. Lallot, Paris, Seuil, 1980, chap. XIII, 1453a30, p. 79.

44. Là encore une recherche par mots clés dans la base de données $I d T$ s'avère particulièrement féconde. Voir par exemple la préface de L'Amour tyrannique de Scudéry, dont Jean-François Sarasin est l'auteur, celle de Scipion de Desmarets de Saint-Sorlin, la dédicace de Clotilde de Jean Prévost, etc. 
II est évident qu'en 1617, quand sont publiées les Tablas poéticas de Cascales, le monde des lettres espagnoles est agité par une polémique féroce autour du théâtre, dont les enjeux sont multiples et au centre de laquelle se trouve Lope de Vega. L'opposition entre Turia et Cascales reproduit celle de Guarini et du Tasse en Italie, dont ils s'inspirent respectivement, ce qui prouve qu'on n'ignore rien en Espagne des débats qui avaient eu lieu en Italie autour du concept de tragicomédie. Si férocement opposés soient-ils, les participants de la controverse trouvent un terrain d'entente à définir la tragicomedia en la rapprochant de la tragedia, davantage que de la comedia. Ce rapprochement est visible dans le traité de Cascales comme chez d'autres auteurs, lettrés ou poètes, et il peut être compris comme le résultat d'un mouvement par lequel le théâtre contemporain se trouve haussé à un niveau de dignité comparable à celui qu'avait la tragédie dans le système classique. Depuis une position assez logiquement inverse de celle de Cascales, l'idée est reprise en 1620 dans la dédicace au dramaturge Guillén de Castro de Las almenas de Toro [Les Créneaux de Toro] par Lope de Vega, qui prouve ne rien ignorer des diverses entreprises de légitimation de la tragi-comédie par les autorités. Malgré sa brièveté (et sa complexité syntaxique), ce texte ramasse les notions fondamentales de la naissance des personnages («personas ») et du style (estilo, qui a dans ce texte un sens passablement imprécis), selon ce qu'exige l'«art » (les règles), tandis que l'usage espagnol est d'exprimer la variété grâce à une formule mixte, qui va « contra el arte » et qui suppose précisément le mélange à la fois de catégories de personnages différentes et du langage dans lequel ils s'expriment respectivement. Mais Lope va plus loin quand il ajoute, avec une allusion aux commencements de l'histoire du théâtre, que la tragi-comédie, du fait de la grandeur (《grandeza ») associée à ses personnages - ou du moins, peut-on supposer, à une partie d'entre eux-, est apparentée, et depuis son origine, à la tragédie. Lope semble désormais assumer, dans une position assez proche en cela des textes de López Pinciano et Cascales cités plus haut, que la tragicomedia est apparentée à la tragédie, plutôt que de revendiquer son indépendance. Quant à créer un terme symétrique, «tragisatiricomédie », pour un mélange du comique et du tragique penchant vers la comédie, cela est inutile, selon Lope, car la comédie présente déjà en elle-même ce mélange des genres ${ }^{45}$.

45. « Dans cette histoire du roi don Sanche, celui-ci apparaît ainsi que d'autres personnages tout aussi dignes de la tragédie, suivant en cela l'usage espagnol, qui veut que l'on mêle, contre les préceptes, les personnages et les styles; c'est pourquoi celui de cette pièce par certains aspects n'est pas très loin de la grandeur mentionnée auparavant, et c'est dans la variété des styles qu'a pris naissance la tragi-comédie. Mais la nouveauté a tant d'éclat qu'en Italie on a ajouté un nouveau terme à ce mélange de styles, alors qu'il a toujours été inclus dans la comédie, et on 


\section{Christophe Couderc}

Ce texte de Lope, daté de 1620 , et quelques autres, pourraient confirmer qu'une évolution du terme de tragicomedia semble bien s'être produite - à moins qu'il ne suffise de parler de palinodie, ou d'incohérence -, depuis un emploi qui pouvait lui donner la même polyvalence que comedia (comme dans l'Arte nuevo, où le terme est implicite, ou l'Apologético, où il est employé par Turia], jusqu'à une acception plus restreinte, qui rattache fortement la tragi-comédie à la polarité tragique du système des genres aristotéliciens. De la sorte, Oleza peut conclure qu'une nouvelle opposition structurerait désormais le système des genres : alors que le théâtre renaissant opposait tragédie et comédie, le théâtre espagnol de l'âge baroque opposerait désormais la tragi-comédie à la comédie ${ }^{46}$. Cette observation est conforme à la volonté qui semble être celle de Lope de Vega quand il désigne explicitement certaines de ses pièces comme des tragicomedias. La question, reprise récemment à nouveaux frais par Florence d'Artois, a fait l'objet d'une étude classique d'Edwin S. Morby, qui concluait à la cohérence de la pratique lopienne de cet «étiquetage » dans la mesure où ses tragicomedias, comme celles de ses pièces qu'il qualifia de tragedias, supposent (ou supposeraient, selon le poète) que le sujet en soit tiré de l'histoire (dans un sens large), tandis que le sujet des comedias serait un sujet inventé ${ }^{47}$. II se trouve que Lope de Vega a assorti un bon nombre de ces tragicomedias de dédicaces dans lesquelles est souligné de façon récurrente, voire systématique, le caractère historique de l'argument de la pièce.

trouve imprimé dans cette langue le titre : tragisatyricomedia! Ce serait là en effet une nouveauté remarquable si la satire, depuis l'époque des Grecs et des Romains, n'était déjà une partie de la comédie » [ « Como en esta historia del rey don Sancho, entra su persona y las demás que son dignas de la tragedia, por la costumbre de España, que tiene ya mezcladas contra el arte las personas y los estilos, no está lejos el [i. e. el estilo] que tiene por algunas partes de la grandeza referida, de cuya variedad tomó principio la tragicomedia. ; Oh gran fuerza de la novedad, pues ya en Italia, a esta mezcla de estilos, se añadió otro, si bien en la comedia estuvo siempre incluso, pues en su lengua anda impresa con este título : tragisatyricomedia, que fuera, como se ve, notable, a no estar la sátira desde el tiempo de griegos y romanos en la comedia ! »] [Lope de Vega, Las almenas de Toro, Dédicace «A don Guillén de Castro »; éd. F. d'Artois, site IdT, NP2-3).

46. « The tragicomedy displaced the need of tragedy due to assuming to a large extent its role concerning the public [...]. The tragicomedy constituted in the end as the modern form of tragedy » (J. Oleza, op. cit., p. 35).

47. E. S. Morby, « Some observations on tragedia and tragicomedia in Lope », Hispanic Review, vol. XI, n³, 1943, p. 185-209 ; F. d'Artois, La Tragedia et son public au Siècle d'or (1575-1635). Introduction à l'étude d'un genre : le cas de Lope de Vega, thèse de doctorat (dir. J. Canavaggio), Université de Paris X, 2009, (à paraître aux Éditions de la Casa de Velázquez), en particulier le chapitre 1. Voir également, pour la question de l'opposition du sujet inventé et du sujet historique, J. Oleza, op. cit. 
On peut en conclure que l'une des raisons pour laquelle Lope choisit l'étiquette de tragi-comédie est que le sujet de ces pièces est partiellement historique mais pas entièrement, comme l'exigerait la pure tragédie, et là serait donc la raison (l'idée était déjà défendue par Morby) pour laquelle le terme de tragedia serait peu employé par Lope. Celui-ci utilise en effet des formulations très proches, quelle que soit par ailleurs l'identité du dédicataire (relation amicale ou possible protecteur ${ }^{48}$ ), pour souligner que l'action de la pièce se fonde principalement sur une « histoire véritable » tandis que la partie inventée, qu'il désigne le plus souvent du terme de «fábula », serait selon lui secondaire ${ }^{49}$. Sommes-nous là face à la définition stable et mûrement réfléchie d'un sous-genre de la part d'un dramaturge inspiré par une longue expérience ? Rien n'est moins sûr, et il convient de contextualiser cet emploi du terme tragicomedia, utilisé dans de nombreux cas, sinon dans tous, à une période au cours de laquelle le poète cherche à s'attirer la faveur des puissants et de possibles mécènes, c'est-à-dire d'une part à se rapprocher du monde courtisan en tâchant de laisser dans l'ombre son activité « mercenaire » de dramaturge fournissant des comedias aux corrales, et d'autre part à mettre en évidence ses talents d'historien : on peut ainsi s'expliquer que Lope réactive l'opposition aristotélicienne entre le poète et l'historien, ou chroniqueur, suivant une stratégie qui lui semble habile au moment où il est animé par l'ambition de se voir confier un poste de chroniqueur royal. La suite des événements a montré que cette stratégie n'avait pas porté les fruits qu'en escomptait Lope, qui n'a jamais pu obtenir cette charge. Tout se passerait donc comme si Lope utilisait désormais tragicomedia non plus comme l'équivalent du générique comedia pour désigner toute pièce de théâtre, mais comme l'équivalent de tragedia, une tragédie mise au goût du jour, conservant de la tragédie la véracité de son sujet mais ne respectant pas d'autres conditions qu'avaient posées la conception médiévale et renaissante. Et il est remarquable de constater que l'opposition de la fable et de la véritable

48. Sur la stratégie d'autopromotion et sur les tentatives (vaines la plupart du temps) de Lope de Vega pour obtenir protections et mécénat, voir dans le présent volume l'article de M. Trambaioli.

49. Par exemple dans la dédicace de El valiente Céspedes, où l'emploi du verbe « lire » indique bien la destination qui est désormais pour Lope celle de son texte dramatique : «que l'on prenne bien garde que dans cette comédie les amours de don Diego sont inventées[.] Cette mise en garde étant faite, on lira les amours de Céspedes comme une invention, mais ses exploits comme une histoire véritable 》 [ «Adviértase que en esta comedia los amores de don Diego son fabulosos [...]. Con este advertimiento se pueden leer sus amores como fábula, y las hazañas de Céspedes como verdadera historia... »] (Lope de Vega, El valiente Céspedes, « Al lector », 1625 ; éd. A. García-Reidy, site IdT, f. $127 r^{\circ}$.] On retrouve des formulations très proches dans les dédicaces de Las grandezas de Alejandro, de El honrado hermano, de Pedro Carbonero, etc. 


\section{Christophe Couderc}

histoire se retrouve sous la plume d'autres auteurs, ce qui peut être interprété comme une preuve de l'impact des moindres écrits du Phénix des Beaux Esprits.

C'est le cas chez Calderón, par exemple, qui commence son activité de dramaturge probablement au début des années 1620. Considéré aujourd'hui communément comme le plus grand auteur de tragédies du Siècle d'or, Calderón n'a sous-titré aucune de ses pièces « tragédie » et n'a laissé aucune considération théorique sur la conception qui pouvait être la sienne des genres dramatiques qu'il a pratiqués pendant plus d'un demi-siècle. II emploie en revanche le terme de tragicomedia dans les derniers vers d'A secreto agravio, secreta venganza $[\grave{A}$ secret affront, secrète vengeance] en insistant sur le fait que son histoire est « véritable »: « ainsi s'achève la véritable histoire du grand don Lope de Almeida, digne objet d'admiration quand termine cette tragi-comédie ${ }^{50} \gg$. Cet unique exemple caldéronien, placé à un lieu du texte quasi paratextuel, semble bien associer l'étiquette de tragi-comédie et le caractère historique du sujet de la pièce, même s'il est possible, comme l'avance Beata Baczynska, que Calderón - qui n'a laissé aucun écrit théorique - manifeste dans ce texte composé au plus tard en 1635, année de la mort de Lope, son opposition (relative) à l'esthétique théâtrale de son illustre aîné. Une sorte de lieu commun, remplaçant celui, plus ancien, qui justifiait par l'exemplarité la peinture des amours lascives de héros condamnés à une fin funeste, se serait ainsi mis en place pour associer à la tragi-comédie la matière historique ; la présence de l'histoire rehausserait ainsi la pièce, et garantirait à la tragicomedia lustre et respectabilité littéraires. Mais si l'on suit sur ce point l'argumentation suggestive de Beata Baczynska, que l'on peut étendre à d'autres pièces de Calderón considérées ordinairement par la critique contemporaine comme des tragédies (par exemple El médico de su honra), il est aussi fort possible de supposer qu'aux yeux de Calderón la construction de l'action de ses comedias tragiques, et en particulier de ses drames de l'honneur (pour proposer d'autres désignations génériques), leur ferait mériter le nom de tragicomedias, si l'on accepte que ce terme désigne, comme l'expliquait Cascales, la tragédie dont Aristote dit que la structure est double : à la différence de la tragédie pure dont le muthos est simple et l'action conduite inexorablement vers son achèvement dans une unique catastrophe hautement pathétique, la tragicomedia, ou tragedia

50. « Esta es verdadera historia / del gran don Lope de Almeida, / dando con su admiración / fin a la tragicomedia ». Le texte de Calderón est cité et glosé par B. Baczynska comme l'élément d’une sorte de controverse, qui reste à étudier, entre Calderón et Lope de Vega : «A Secreto Agravio, secreta venganza y el debate sobre la "tragedia española" », dans M. Tietz (dir.), Calderón y el pensamiento ideológico y cultural de su época, XIV coloquio anglogermano sobre Calderón, Stuttgart, F. Steiner, 2008, p. 31-45. 
double, s'achève par un dénouement construit en deux temps, ou présentant deux facettes, l'une positive et l'autre négative, et éveille de ce fait chez le spectateur les émotions de la tragédie et celles de la comédie ${ }^{51}$.

Les opinions d'autres auteurs, principalement des lettrés (davantage que des poètes et des praticiens de la scène), pourraient être convoquées afin de contribuer à la définition de la tragicomedia dans le contexte du théâtre espagnol du Siècle d'or ; mais on observerait alors qu'à partir d'une série assez limitée de traits définitoires, les variations peuvent être assez nombreuses. II n'y a rien d'étonnant à cela, dans la mesure où le terme de tragicomedia, créé par hybridation, est en quelque sorte par nature rétif à une définition essentialiste et stable. Au-delà de cette remarque générale, l'intérêt de l'étude des paratextes, dans le cas espagnol, est confirmé par la proximité qui s'y laisse voir entre la pratique et la théorie, contrebalancée par le fait que dans de nombreux cas le paratexte dispose d'une autonomie parfois très grande par rapport au texte représenté. De ce point de vue, il n'y a donc rien d'étonnant à ce que la définition que recouvre le terme évolue en fonction du contexte dans lequel elle est convoquée. La remarque vaut naturellement en premier lieu pour Lope de Vega, qui est à la fois l'auteur dramatique le plus prolifique de son temps, mais également le dramaturge qui nous a laissé un corpus paratextuel de première importance, dans lequel il est tentant de rechercher l'expression d'une pensée cohérente ; or on voit, en particulier chez cet auteur, comment le terme tragicomedia est aisément objet de manipulation ou d'instrumentalisation à des fins purement tactiques et conjoncturelles. À cette observation concernant le caractère asystématique des définitions proposées, parfois par les mêmes auteurs ${ }^{52}$, il faut enfin ajouter que la porosité semble toute relative, en ce qui concerne le contexte espagnol, entre les idées des doctes et celles des praticiens. Plus précisément - mais cette question mériterait d'autres développements -, le terme de tragicomedia, objet de débats et de polémiques, a

51. Voir B. Baczynska, ibid., ainsi que C. Couderc, Le Théâtre tragique [...], éd. cit., p. 77 sq.

52. Sur ce point, le riche paratexte qui accompagne par exemple la tragicomedia Los jardines y campos sabeos de F. Enríquez de Guzmán mériterait un examen particulier, rendu possible par le travail d'édition dans le cadre du projet $I d T$ : se revendiquant comme une imitatrice des modèles anciens («imitando a los cómicos antiguos, / [nuestra poeta] propiedad ha guardado, arte y preceptos / de la antigua comedia », F. Enríquez de Guzmán, Tragicomedia Los jardines y campos sabeos, « Prólogo », 1624 ; éd. E. Villegas de la Torre, site IdT, v. 37-39), l'auteure désigne alternativement sa pièce comme tragicomedia et comme comedia, et elle n'explicite que dans un prologue ajouté lors d'une seconde édition (1627), imprimée trois ans après la première, le choix du terme tragicomedia par référence au concept du « mixte », comme par exemple avant elle Ricardo de Turia que nous citions plus haut. 


\section{Christophe Couderc}

bien semblé apte à de nombreux lettrés pour désigner tout ou partie des formes dramatiques en usage à cette époque. En définitive tout se passerait comme si le terme tragicomedia avait été accepté, quoique de façon différente, sur le plan spéculatif, comme une catégorie abstraite utile à la pensée du genre ${ }^{53}$, mais que, paradoxalement, le terme comedia, étiquette hypergénérique, devenait au même moment hégémonique pour désigner toute œuvre théâtrale.

Christophe Couderc Université Paris Ouest Nanterre La Défense

EA 369 Études Romanes

53. Voir par exemple les témoignages rassemblés par I. Arellano dans « Lo trágico y lo cómico mezclado », Rilce, vol. 27, n¹, 2011, p. 9-34. Voir également, du même, « Cuestiones taxonómicas y tragedias calderonianas », dans C. Couderc et H. Tropé (dir.), La Tragédie espagnole et son contexte européen (XVI ${ }^{e}$-XVII ${ }^{e}$ siècles), Paris, PSN, 2013, p. 249-256, notamment p. 251-253. 